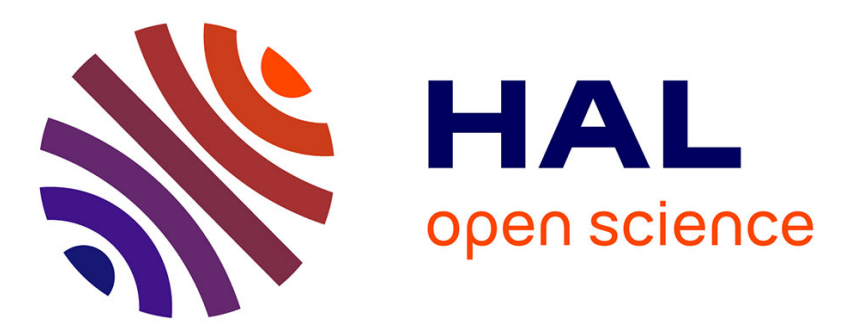

\title{
Sowing the seeds of algebraic generalization: designing epistemic affordances for an intelligent microworld
}

Manolis Mavrikis, Richard Noss, Celia Hoyles, Eirini Geraniou

\section{To cite this version:}

Manolis Mavrikis, Richard Noss, Celia Hoyles, Eirini Geraniou. Sowing the seeds of algebraic generalization: designing epistemic affordances for an intelligent microworld. Journal of Computer Assisted Learning, 2012, -, 17 p. hal-00704034

\section{HAL Id: hal-00704034 \\ https://hal.science/hal-00704034}

Submitted on 4 Jun 2012

HAL is a multi-disciplinary open access archive for the deposit and dissemination of scientific research documents, whether they are published or not. The documents may come from teaching and research institutions in France or abroad, or from public or private research centers.
L'archive ouverte pluridisciplinaire $\mathbf{H A L}$, est destinée au dépôt et à la diffusion de documents scientifiques de niveau recherche, publiés ou non, émanant des établissements d'enseignement et de recherche français ou étrangers, des laboratoires publics ou privés. 


\title{
Sowing the seeds of algebraic generalization: designing epistemic affordances for an intelligent microworld
}

\author{
M. Mavrikis, R. Noss, C. Hoyles \& E. Geraniou \\ London Knowledge Lab, Institute of Education, University of London, London, UK
}

\begin{abstract}
This paper describes the design of a mathematical microworld to tackle the persistent difficulties that secondary school students have with the idea of algebraic generalization, which is a key stumbling block in secondary-school mathematics classrooms. Our focus is to characterize algebraic ways of thinking and to design both affordances of the system as well as suitable tasks and pedagogies that provide a substrate of activity and experience for the teaching and learning of algebraic generalizations. Using as reference illustrative cases of 12 to 13-year-old students' interaction with the microworld, we demonstrate the strong interplay between epistemology and the design of the microworld and draw conclusions regarding its potential to support the development of algebraic ways of thinking.
\end{abstract}

Keywords design, epistemic affordances, intelligent support, microworlds.

\section{Introduction}

Our interest focuses on the epistemological dimension of the design of exploratory digital learning environments, specifically mathematical microworlds. In mathematical microworlds, students engage with some mathematical concepts not only by exploring the objects in the microworld, but also by building their own while making explicit the mathematical relationships between and within the objects (Thompson 1987; Hoyles 1993). Although it is possible to develop microworlds that afford the opportunity for students to express and reflect on their mathematical thinking with teachers drawing students' attention to crucial aspects of the tasks at hand (Noss \& Hoyles 1996), we have argued elsewhere (Hoyles et al. 2004) that there remains a need to elaborate the kinds of mathematical knowledge that students develop during such microworld interactions.

We are inspired by Papert's argument of over 40 years ago, that students need to develop 'Mathematical Ways of Thinking', while recognizing that these 'can only be taught by using particular topics as vehicles' (Papert 1972, p. 251). Similarly, more 
recent ideas are set out by Cuoco et al (1996) in describing the development of mathematical habits that can start in early years and, if nurtured, can serve the learning of 'formal' mathematical topics (Goldenberg et al. 2010). While mathematical microworlds make the objective of fostering these habits of mind possible, our view is that (at least) three epistemological challenges must be addressed by designers to achieve this objective. These are: first, to characterize the mathematical way of thinking in question; second, to design corresponding affordances in the microworld and appropriate accompanying activities; and third, to map out the way that potential interactions in the microworld support the development of these ways of thinking.

This paper presents our attempt to deal with these challenges in the context of a 3-year research programme, the MiGen project. The MiGen project set out to design a pedagogical and technical system to support students in developing a propensity to strive for algebraic generalization, a particular and fundamental mathematical way of thinking. At the core of the MiGen system is a mathematical microworld, the eXpresser, in which students can construct figural patterns using coloured square tiles. However, underlying the surface goal of building patterns is our main objective; namely, that through interaction with the system, students develop an appreciation of the idea of algebraic generalization. Of course, in interacting with the microworld, we anticipated that students would also engage in more general mathematical ways of thinking, such as being an'experimenter', 'visualiser', and 'describer' (Cuoco et al. 1996; Goldenberg et al. 2010). Students will also bring to bear a range of problem-solving heuristics, such as analysing situations, interpreting a problem, and other strategies described by Polya and later by Schoenfeld (1985). To these we include developing ownership over the solution process and being able to defend and justify it (cf. Küchemann \& Hoyles 2009). However, our main research endeavour concerns how to design a software and activity system that sow the seed of algebraic generalization and serve as vehicles for fostering its development. The section 'Algebraic ways of thinking' presents a brief epistemological analysis of algebraic generalization from which we derived the following three key 'algebraic ways of thinking' that underpin the idea of generalization:

1. Perceiving structure and exploiting its power;

2. Seeing the general in the particular, including identifying variants and invariants;

3. Recognizing and articulating generalizations, including expressing them symbolically.

The 'MiGen project' section presents the design methodology and a short description of the MiGen system including the eXpresser, and accompanying activities. The rest of the paper provides vignettes derived from illustrative student interactions with the system that demonstrate the trajectory by which students develop these ways of thinking and the last Section of the paper provides a summary and conclusions outlining future challenges that are shaping our immediate lines of research. 


\section{Algebraic ways of thinking}

We now turn to a brief summary of the arguments leading to the algebraic ways of thinking, which underpin our design of the eXpresser.

\section{Perceiving structure and exploiting its power}

At a general level, we build on the work of Cuoco et al. (1996) who have suggested that algebraists have a tendency to express structure in different but connected representations. To do this requires an algebraic way of thinking that involves insight into the mathematical structure of a problem situation, and an orientation to structural rather than merely empirical reasoning (Küchemann \& Hoyles 2009). There exists a substantial body of research that demonstrates an approach to developing algebraic thinking through figural pattern- making (Lee 1996; Mason 1996; Küchemann 2008; Rivera 2010). In our view, the potential of this approach stems from the motivation it affords for developing structural reasoning and the habits of 'breaking things into parts' by identifying 'the building blocks of a structure' (Cuoco et al. 1996).

There are numerous examples in the literature that demonstrate that these habits are not as simple as one might expect (Küchemann 2008; Rivera 2010). In particular, Küchemann (2010) provides an extensive review and discussion of the complex interplay of current curriculum practices, students' (and often teachers') lack of awareness of the benefit of looking for structure, and the nature of the tasks' characteristics and presentation (cf. also Chua et al. 2009). All too often, students are encouraged to justify perceived patterns by a standard sequence of numerical procedures such as number-pattern-spotting, which tend to take the place of any structural reasoning (cf. Healy \& Hoyles 2000).

The tension in a school situation is that students are inevitably focused on task completion, bypassing any need to mobilize structural reasoning or algebraic generalization. Of course, injudiciously designed tasks can easily compound the difficulty. An example of the tension is characterized by the need (often only apparent to the teacher) to generalize from particular cases with questions such as 'How many here?', 'How many there?', and 'How many in general?'. Here lies the first epistemological obstacle. The teacher's epistemology (i.e. the special case is merely a way of thinking about the general case) is at odds with the student's (i.e. I am answering all the special cases right, what is this totally other thing the teacher wants to know - and why?). Resolving this tension is by no means a trivial process, and unlikely to happen unless there is an opportunity for identifying structure, and an advantage in exploiting it (Redden 1996; Healy \& Hoyles 2000; Radford 2001). 


\section{Seeing the general in the particular, including identifying variants and invariants}

In the pursuit of further ways of thinking that pertain to algebraic generalization, we were also inspired by proponents of approaches to algebra such as Mason (1996) and Love (1986), who describe 'seeing the general in the particular' as the core of generalization. In particular, Mason et al. (2005) describe a pedagogical construct, essentially a way of thinking, to which they refer as manipulating-getting-a-sense-ofarticulating. This triadic process recognizes that by manipulating a special and familiar case one can 'get a sense of' and eventually articulate (not necessarily in standard symbolic form) what stays the same and what changes (cf. Lobato et al. 2003; Becker \& Rivera 2008). For experts and novices alike, this is not always straightforward.

In a recent literature review, Dörfler (2008) revisits the types of generalization and argues that a constructive process requires actions that allow variant and invariant relations to emerge. Related research (e.g. Pellegrino \& Glaser 1982; Greeno 1991; Sutherland \& Rojano 1993; Rojano 1996; Haverty et al. 2000; Nunes \& Bryant 2010) also indicates that the process of algebraic generalization requires a general propensity to recognize a relationship between quantities and variables and 'to express this recognition using general statements' (Thompson 1993).

\section{Recognizing and articulating generalizations, including expressing them symbolically}

Dörfler also argues that the emergence of variants and invariants plays an important role in the crucial step of symbolization. Thus, the availability of symbols that can play an expressive role is essential. The need to develop a symbolic system for expression has occupied us in the past (Noss et al. 1997). Kieran (1989) also argued that a necessary component of algebraic thinking is the 'use and availability of symbolism to reason about and express generalisations' (Kieran 1989), and a similar argument has been made more recently by Carraher et al. (2008).

The literature, therefore, helped us converge on a single overarching challenge: How can we design a microworld that incorporates a symbolic system for the expression of relationships? Clearly this had better not be the conventional symbol system of algebra - that is what we are trying to teach! How can we ensure that the system has some of the power of the x's and y's of conventional algebra, but which is more finely tuned to the emerging abilities of students, who are coming to understand the power of structure, but do not yet know how to express it? 


\section{The MiGen project}

The MiGen project aimed to co-design with teachers, and build and evaluate an intelligent learning environment for supporting the development of students' algebraic generalization through building tiling patterns. We recognized from the outset that the role of the teacher as a 'facilitator' or 'orchestrator' is hugely demanding (Hoyles \& Sutherland 1989; Trouche 2004). Thus, a further goal was to develop an intelligent subsystem to support both the students directly, and to provide information to teachers that assists them in their classroom role. This intelligent component of our system is outside the scope of this paper, although it does feature in one vignette. For the design and details on the intelligent subsystem see Mavrikis and Gutierrez-Santos (2010) and Gutierrez-Santos et al. (2010). For early prototypes of the tools that can assist the teacher see Gutierrez- Santos et al. (in press).

\section{Methodology}

As Pirolli and Greeno (1988) put it, as designers of the eXpresser microworld at the heart of the MiGen system, we had to engage in a search process over the space of possible designs. Crucially, we had to revisit our epistemological orientations and come to grips with the nature of the knowledge we expected students to construct in interaction with our microworld (diSessa1995). This was an iterative process, in which theory development, design, and experimentation with the emerging software proceeded hand in hand.

Our methodology has evolved in a series of iterative cycles interleaving software development with experiments with groups of students and teachers in both the laboratory and in classrooms. In the past 3 years, and with successive versions of eXpresser, we have conducted several one-to-one, small-scale, and whole- classroom studies. In particular, at the time of this writing, more than 200 students (11-13 years old) have interacted with expresser. Examples of these iterations and students' interactions are reported in Geraniou et al. (2009a) and Noss et al. (2009). These design experiments also allowed us to develop explicit pedagogical strategies that could be followed by the teacher (with the support of teacher tools) or delegated to the intelligent system, as appropriate (see Mavrikis et al. 2008; Geraniou et al. 2009b; Mavrikis and Gutierrez- Santos 2010).

As the reader will appreciate, our understanding of student learning outcomes evolved, as we gained more data during the iterative cycles of design and evaluation. In this paper, we focus not on learning outcomes but on design, and a particular stable iteration of the system that was used in school studies with three classes of 12 to 13year old students in three different London schools (varying from 16 to 25 students each). In what follows, we first describe the version of expresser and the 
accompanying activities to help the reader gain an appreciation of the environment. ${ }^{1}$

'Student vignettes using eXpresser' present illustrative examples that demonstrate the interplay between students' interaction in eXpresser and the ways of thinking that were outlined in the previous section.

\section{The eXpresser}

In eXpresser, students are presented with a model and asked to construct it using one or more patterns (see Fig 1). The model is animated, with the Model Number changing randomly. The animation serves to emphasize the generality expected: i.e. the task is not to count the tiles. Rather, it is to find a rule that would give the number of tiles for any given model number (see Noss et al. 2009 where this is discussed in more detail). Having been set the task, students are required to build the model and the general rules that govern it. The students set about constructing the model, first by expressing how they visualize its structure as sets of patterns. Each pattern takes the form of repeated building blocks that are appropriately placed on the 'canvas'.

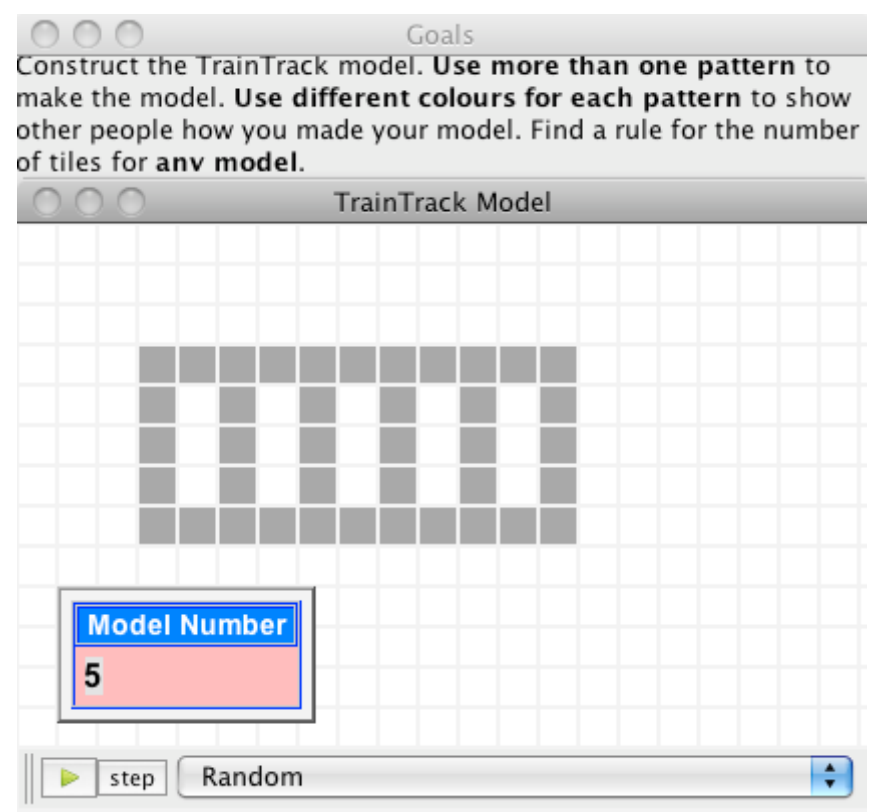

Figure 1 The 'Train Track' task in eXpresser. The model to be constructed is animated with the 'Model Number' (the number of 'holes' in this case) changing in random steps every few seconds

Students then make explicit their rules to calculate the number of tiles in each pattern. When the rule is correct, the pattern becomes coloured. Finally, students are encouraged to use their rules to obtain the total number of tiles needed in the model: the sum of the tiles needed for each of the constituent patterns. Of course, there is always more than one way to do this. 
Figure 2 shows a snapshot of the eXpresser with the Train Track task of Fig 1 completed. 'My Model' has been built on the left canvas this time by combining two patterns, one coloured green and the other red. (We will refer to 'red' and 'green' throughout the paper. In grey-scale, these colours appear as dark and light grey, respectively). A pattern in eXpresser comprises a repeated element, called a 'building block' (shown in A), which is created by grouping several tiles together. It is worth noting that single tiles or whole patterns can also be considered building blocks themselves, thus leading to complex patterns of patterns.

When making a pattern, students have to specify the translations across and down for each repetition of the building block, as well as an initial number of repetitions. In Fig 2, a $\mathrm{C}$-shaped building block $(\mathrm{A})$ has been created and it is repeated by placing each repetition two squares across and zero places down. ${ }^{2}$ When the C-shaped building block (A) is made, its properties are shown in an expression (B). In Fig 2, the building block is repeated as many times as the value of a variable called 'Model Number' (D), in this case 4. As students build their constructions in 'My Model', a second canvas is seen alongside (the 'General Model'). This mirrors exactly My Model until the student has introduced a variable into their model (how this is achieved is presented in more detail in the next Section).

Patterns will be coloured by calculating and then allocating the exact number of coloured tiles to its construction. In the case of the pattern made of $\mathrm{C}$-shapes, using the expression for construction $(B)$ and the number of times the building block is repeated, the rule for the total number of tiles in the green pattern is 'Model Number $X 7$ ', in Fig 2e. When variables have been introduced in My Model, eXpresser will randomly change their value in the General Model. In Fig 2, the value of the Model Number in the General Model is randomly set to 9 , resulting in a different instance of the model $(F)$. The General Model is coloured only when students express correct general rules in the 'Model Rule' area of the screen $(G)$. 


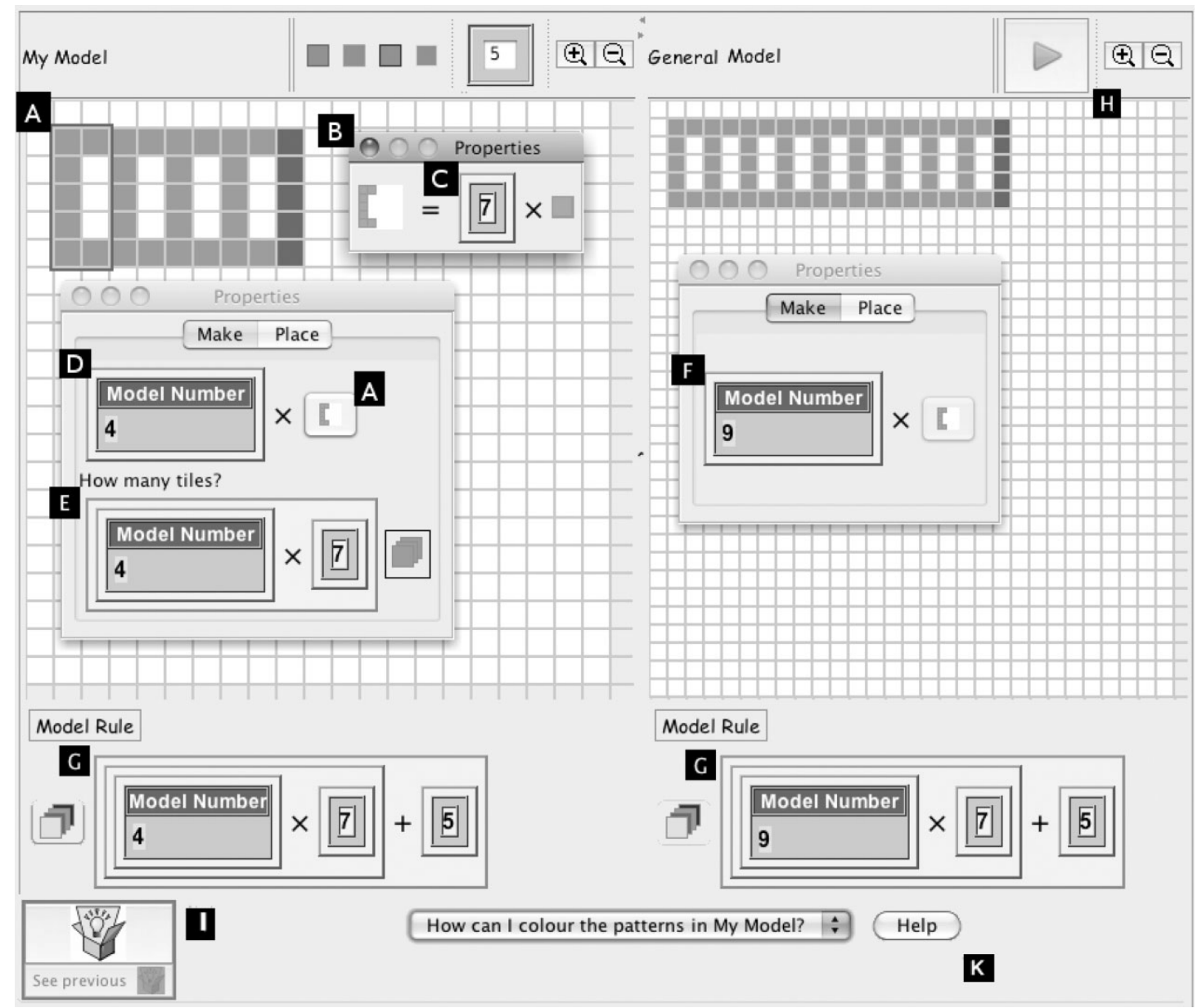

Figure 2 The main interface of eXpresser. (a) Building block to be repeated to make a pattern. (b) Expression of construction of the building block. (c) The number of tiles of the building block. (d) The number of repetitions of the building block in this case 4, i.e. the value of the 'variable' 'Model Number'. (e) Number of tiles required for the pattern, with general rule. (f) Any variable used in 'My Model' takes a random value in the 'General Model'. (g) For the General Model to be coloured a general rule is required that expresses the total number of tiles in the whole model. (h) Patterns can be animated using the play button, which randomly sets the value of the variables in the General Model, with the model remaining accurate and coloured if and only if the rule is correct. (i) A suggestion button lights up to provide support to students. (k) A drop-down menu that allows students to choose a sentence and ask for help

Students cannot interact directly with the General Model. They are, however, encouraged (by the design of the activity and by system prompts) to click the play button $(\mathrm{H})$ to animate their model to test its generality. To support students during their interaction, the microworld also has a suggestion button (Fig 2i), which only lights up if the system observes an action that implies that help is warranted. Rather than interrupting the student directly, the icon simply lights up to indicate that a suggestion is available: the students are free to ignore these suggestions or evaluate and follow them. The other component (Fig 2k) comprises a drop-down menu that allows students to choose a sentence and ask for help. This provides the system with an indication of the student's need, and an opportunity for the student to reflect on what they are trying to do by engaging further with the discourse of eXpresser. 


\section{Student vignettes using eXpresser}

This section illustrates the affordances that underpin the design of eXpresser in the light of our characterization of algebraic generalization, and illustrates some students' evolving algebraic ways of thinking. We refer to these microworlds' affordances as 'epistemic' to differentiate them from 'action' or 'pragmatic' affordances that are relatively independent of any epistemic basis. Rather like Gibson's (1977) view of affordances as preconditions for activity, we view epistemic affordances as preconditions for learning - in our case allowing the students to develop the three algebraic ways of thinking we focus on this paper. Of course, we take it for granted that the embedding of affordances into software may be a necessary but a far-fromsufficient condition for learning to take place.

The data in this section are derived from the school studies mentioned on page 5 . We focus on the cases of five students, Alicia, Conor, Fiona, Greg, and Maria (not their real names), who are illustrative of possible students' constructions and interactions in eXpresser and of the answers they provided in pre- and post-tasks. Our rationale for which data to report is to demonstrate the potential of eXpresser in supporting the development of the kind of algebraic thinking in question. In each one of the studies concerned, the teacher introduced expresser in the classroom through a series of structured and guided tasks. Each student was working individually on a computer, with the teacher available as necessary. In the following session, students used eXpresser to build simple patterns, and in the third session they were asked to undertake the Train Track task outlined earlier (see Fig 1). While the students were working, one or two researchers observed the class acting as teacher assistants or providing technical support. Together with the teacher (and mostly responding to her requests) they took on the role of occasional facilitators, supporting students when needed.

After constructing their model for Train Track and expressing the rules in eXpresser, students were given off-computer tasks (we will call them post-tasks) that were designed to provoke them to reflect on their activity and make visible the algebraic ways of thinking they may have developed. Examples are shown in Fig 3.

\section{Perceiving and exploiting structure}

When students are presented with a model and a task description by the system, they are encouraged to think about its structure in terms of its constituent patterns, in order to construct it. The choice of the patterns and the structure of the model are left to the students who are asked to depict its structure using different colours. Early sessions with eXpresser showed that students tend to visualize the same models very differently, leading to different conceptualizations of structure and rules. Table 1 shows some of the different ways in which students chose to construct the 'Train Track' 
pattern.

Alternative ways of seeing and expressing the relationships serve in our activity sequence as a basis for potentially fruitful discussions between students, giving them the opportunity to appreciate the qualities of different models in terms of how complex, systematic, or general they are (cf. Geraniou et al. 2010).

As we explained earlier, an important step in building a pattern in eXpresser is the identification of a building block, i.e. a unit of repeat. Although it is possible for students to drop single tiles and 'draw' a pattern rather than identify its building blocks, this strategy is soon found to be problematic, as constructing a general model and animating it using the play button is not possible.

(a)

For the pattern of Figure Number 4 below, you need 27 tiles

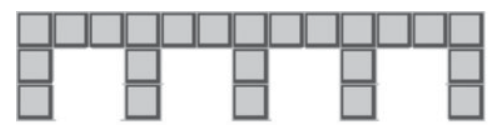

a. How many tiles do you need to build the pattern of Figure Number 5 ? b. How many tiles do you need to build the pattern of Figure Number 10 ? c. How many tiles do you need to build the pattern of Figure Number 100 ? d. Write down a rule for the number of tiles for the pattern for any Figure Number. Show how you worked out your answers.

(b)

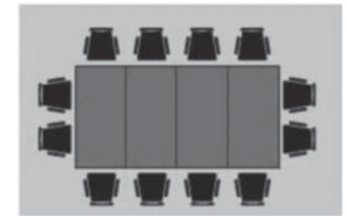

4 tables

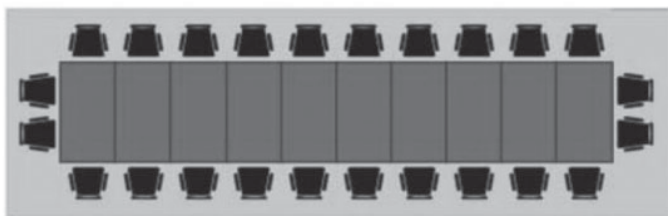

10 tables
Figure 3 Sample post-tasks designed to probe students' thinking after working with eXpresser. (a) Bridges. (b) Tables and chairs. tables.

1. Find the general rule for the number of chairs for any number of

2. Use your rule to find the number of chairs for 20 tables.

3. Use your rule to find the number of chairs for 200 tables.

4. If I have 26 chairs, how many tables do I need?

We have presented elsewhere strategies that exploit the notion of the 'rhythm of action', to help students identify building blocks. In brief, this involves drawing students' attention to the patterns they are implicitly repeating while dropping tiles (see Mason et al. 2005; Radford et al. 2006; Noss et al. 2009).

Although we did not have a control group of students working on the post-tasks, we know from our own previous studies and the relevant literature (Kieran 1989; Mason 1996) that students of this age (12-13 years old) usually resort to counting in order to answer 'How many' questions - it is, after all, rather natural to answer a 'how many' 
question by counting, especially if the thing being counted is static! Our preliminary evidence indicates that the epistemic affordance of turning groups of tiles into building blocks, which then enables the students to construct a pattern with feedback as to its accuracy, contributes to students adopting a structural approach rather than relying on counting.

First, we saw that during interaction with eXpresser, there was a clear tendency to at least recognize (but not necessarily express symbolically) that the total number of tiles needed for a pattern can be found by multiplying the number of tiles in the pattern's building block by the number of times the block is repeated.

Second, in the pencil-and-paper setting before the eXpresser work, we found that all of the students of our illustrative cases in questions similar to (a) of Fig 4 chose just to draw the configuration and count. However, after interacting with eXpresser, they demonstrated an awareness of the structural coherence that underpins these figural patterns and showed a tendency to coordinate the identification of structure with its symbolic representation. We provide two examples in Fig 4, Fiona and Alicia's diagrammatic annotation of their answer in the Bridges and Tables post-tasks in Fig 3. These demonstrate that instead of relying on counting, the two students identified building blocks in the figure enabling them to see the structure of the problem.

When Fiona (Fig 4a) was asked why she circled the two groups of tiles in the pattern, she explained how she would use eXpresser to solve this problem by first constructing the two building blocks. The written answer that is struck through $(6 \times 4+3=27)$ shows that even to determine the number of tiles for Figure Number 4 (which was already provided for her), Fiona used a structural approach to show how the result can be calculated (albeit in a typical 'loose' notation - cf. Küchemann 2008) - rather than counting the tiles.

When showing how she worked out her answer, the schematic drawing demonstrates an attention to structure, even in answering a question that does not strictly encourage it (she could after all just draw Figure Number 5 and count the tiles). Alicia's answer (Fig 4b) is similarly structural indicating that she built on the residue of her interaction with the eXpresser. 
Table 1 Different conceptualizations of the structure of Train Track model

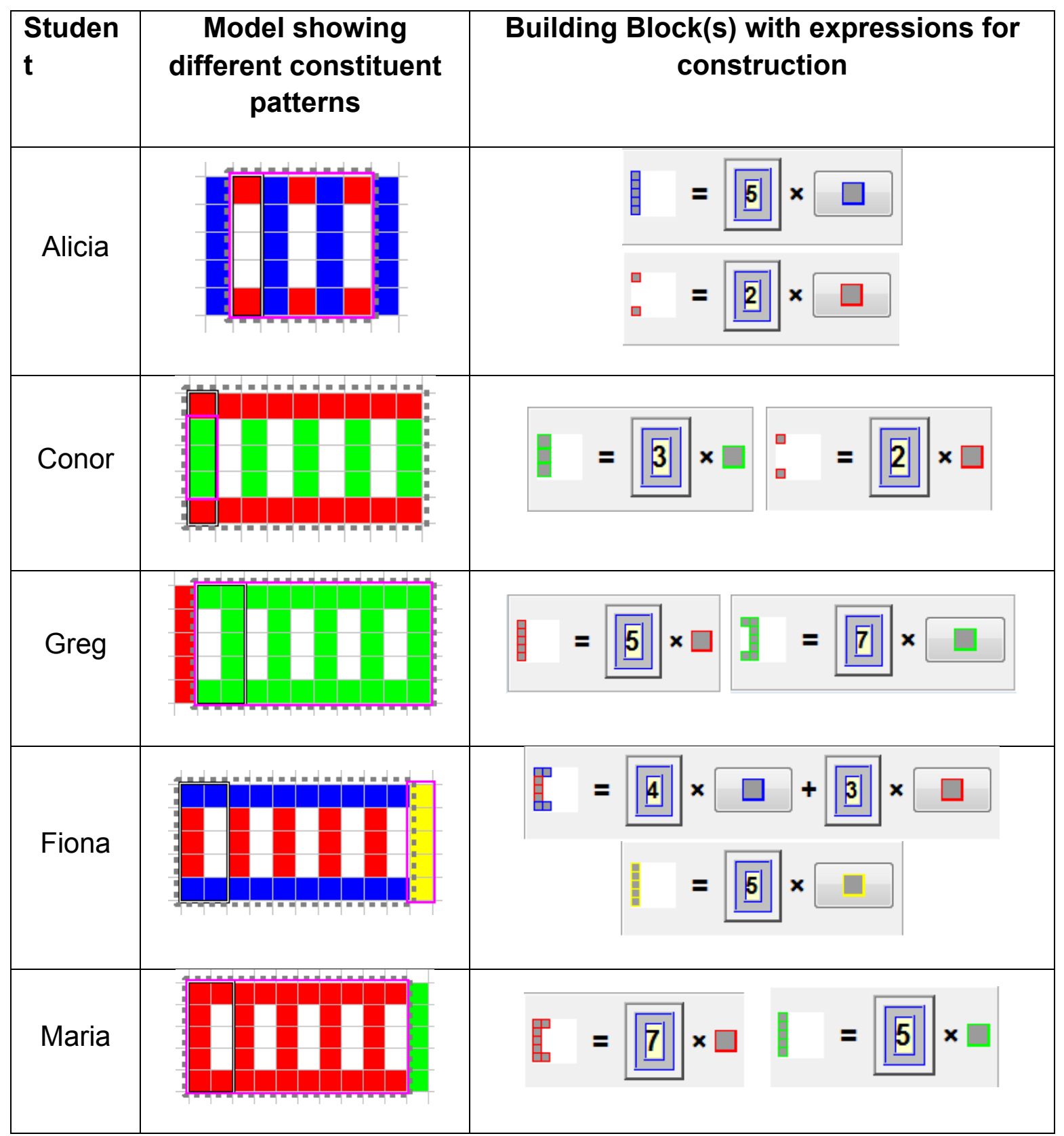

Seeing the general in the particular: identifying variants and invariants

Having constructed a model, students are challenged to identify variants and invariants of their model. As presented on page 4, this is an important stage in generalization. It is achieved in eXpresser by 'unlocking' the numbers that have been used to specify the properties of their model. These are, by default, 'locked'.3 An unlocked number is 'free' 
(unlocked from having a single value) and takes a randomly generated value in the General Model. The General Model area and the play button have a crucial role at this point: they provide the rationale for unlocking numbers, and provide feedback as to the outcome of unlocking.

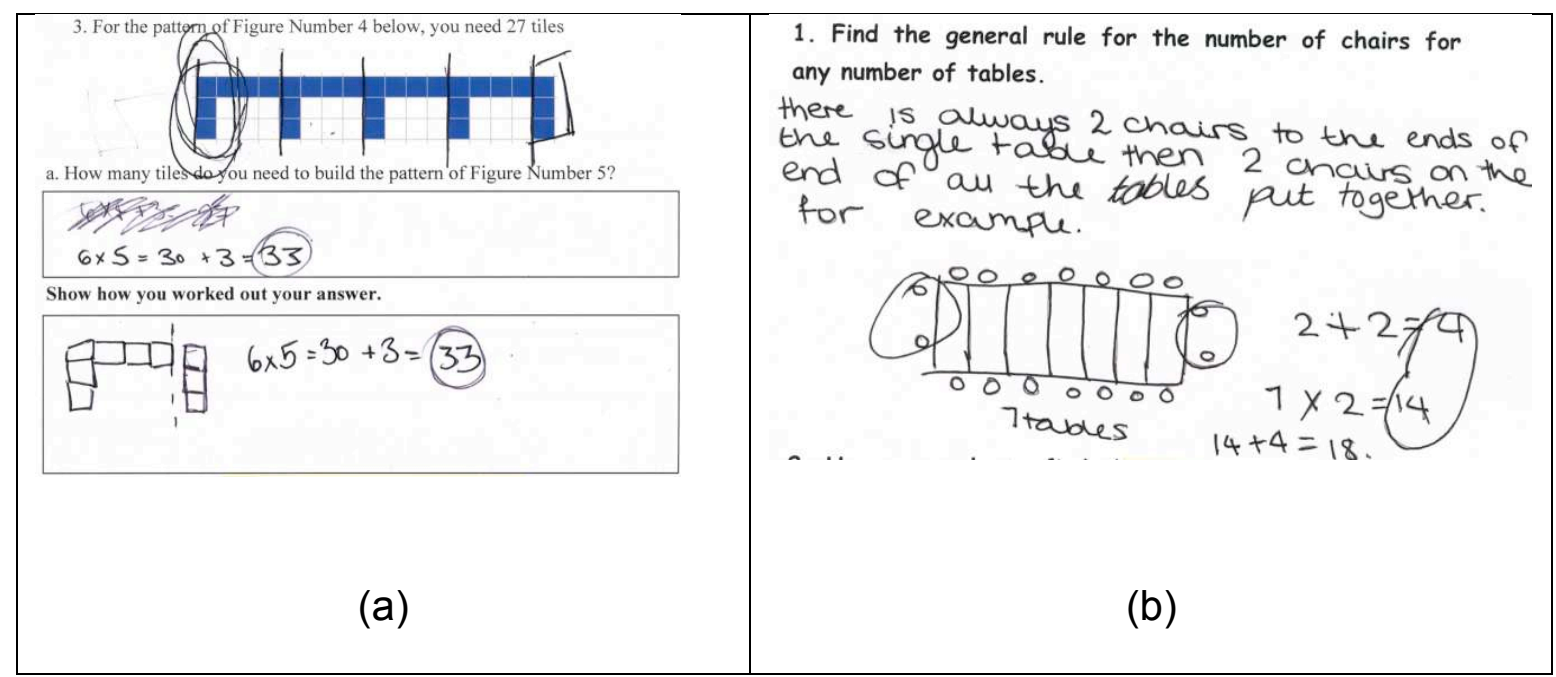

Figure 4 Sample answers to post-task questions. Both Fiona (a) and Alicia (b) have identified building blocks, which have helped them take a structural approach in finding a general rule.

It is here that it becomes apparent that considering the epistemic affordances of the environment independently of context is not sufficient. The actual motivation for generality is provided by the main goal of the task, i.e. to produce a model that will animate correctly by colouring the exact number of tiles required, in combination with a pedagogical strategy that challenges students to construct models that are impervious to changing values of the various properties of their construction. Healy et al. (1994), inspired by a term students employed, refer to this strategy as 'messing-up'. Its success in previous work in dynamic geometry led us to hypothesize that it would be effective in our case. In expresser, this strategy takes several forms. First, it is introduced in the classroom culture by the teacher in introductory activities. Second, it is instantiated in the General Model that demonstrates if the construction is structurally correct or not for the different values of the unlocked numbers. Third, as we will see more clearly in the next section, the intelligent subsystem is designed to draw attention to any lack of generality and, if it exists, to provide feedback by messing up the student's model (My Model).

Thus, unlocking a number becomes a crucial and necessary step. If the numbers remain locked, the General Model looks exactly the same as the specific model and will not animate. If this happens, and is drawn to their attention by the intelligent support, students often unlock numbers without much consideration of the impact of their action. However, at this point, we have found that the visual feedback provided by the General 
Model helps students become aware of the results of unlocking, and thus begin to make sense of the role of variants and invariants in their model.

For example, Greg unlocked several numbers unnecessarily (perhaps we could call this the 'unlock- everything-to-be-on-the-safe-side' strategy). Yet after having his attention drawn to the General Model by the intelligent subsystem, he locked the numbers again. By guess and test, he realized that the important number to unlock was the one used to specify the number of repetitions. However, he unlocked the number of repetitions in both patterns, that is the 5 (vertical column on left) as well as the 6 (C's). Prompted by the system, Greg clicked the play button and noticed how the General Model was 'messed up' (see Fig 5). Eventually, Greg locked the 5, the number of repetitions of the red pattern, thus recognizing explicitly that the number of repetitions of the C's was the key variant.

We have observed other students following the same strategy. More generally, we have seen how the task goals and the feedback from messing up provide incentives for students to observe and analyse the outcomes of their actions and, last but not least, to distinguish variant and invariants that emerge directly from their own actions. It is through these actions that students begin to bring together the structural and the symbolic representations.

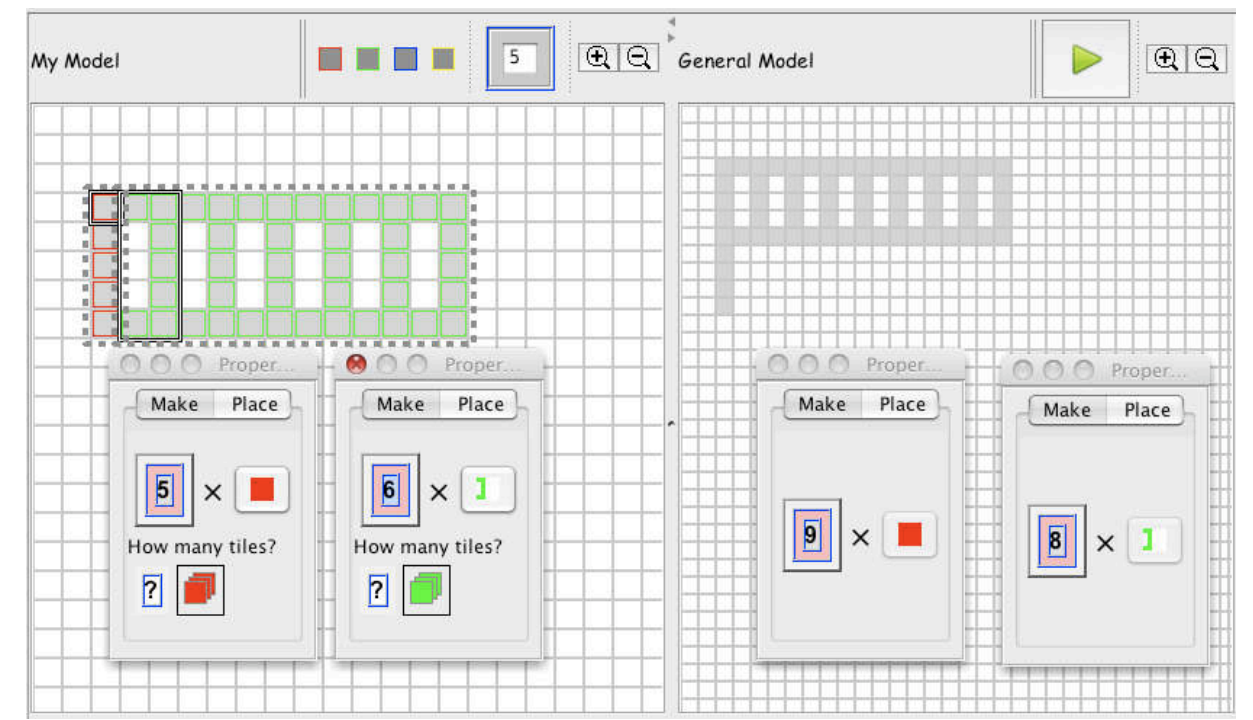

Figure 5 Greg's model. Unlocking the 5 messes up the model in the General World

\section{Recognizing and articulating generalizations symbolically}

Noticing the structure of patterns in a model and constructing it is relatively straightforward for students compared with recognizing relationships within and between patterns and making them explicit in order to express the total number of tiles 
required. Unless some motivation is provided, students - quite naturally - fail to see the need to recognize and articulate relationships in a general way, let alone express them symbolically.

Colouring a pattern - expressing relationships between quantities within patterns As already mentioned, it is possible to find the number of tiles needed to colour a pattern, either by simply counting or by implicitly noticing the relationship between the number of tiles in a block, the number of its repetitions, and the total number of tiles needed to colour the pattern. The difficulty, however, is to articulate what this relationship is, and to express it in a general way.

We described on the previous page how the goal of making models animate is linked to the unlocking of numbers. Once students unlock a number, the rationale for constructing a general statement about the colouring of the pattern tends to become clear. The classroom culture of encouraging 'messing-up' (see page 12) in combination with the support from the system, provides an incentive for students to analyse and express a rule for the number of tiles in the pattern in a general way.

Let us demonstrate this through Greg's actions in one of the introductory tasks. The snapshots in Fig 6 demonstrate a typical interaction that provides Greg the incentives and opportunity to analyse and express the generality that he perceived (he could provide the right answer) but found it hard to express. Before the vignette, Greg was already familiar with the eXpresser and had coloured patterns generally with the help of the teacher demonstrating it in the classroom. Nevertheless, he still initially failed to connect an expression with the structure of the model (Fig 6a). The beginning of his interaction is rather typical of ways of thinking that are still some way from anything we could describe as algebraic. We could observe him repeatedly counting and providing the number of tiles in the pattern (Fig $6 \mathrm{~b}-\mathrm{e}$ ), but only after the system's suggestions (Fig 6e-g) that drew his attention to the need to link the two quantities, Greg created an expression that according to him (revoicing the teacher's words in an earlier session) 'tells it [the eXpresser] how many tiles it needs for any number in the pink [unlocked] box'. 


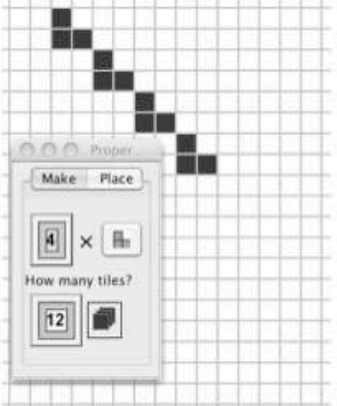

(a)

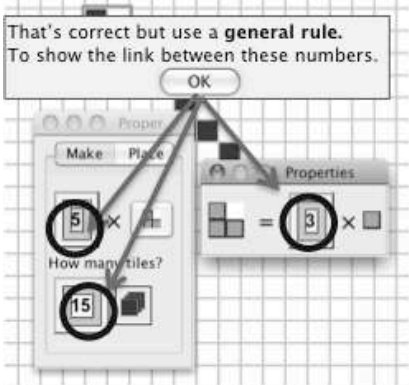

(e)

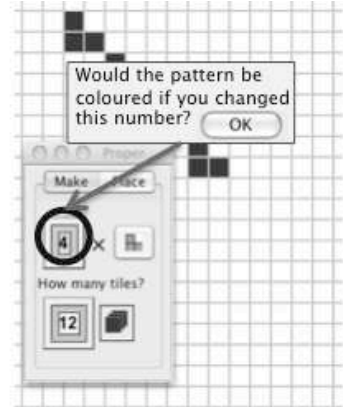

(b)

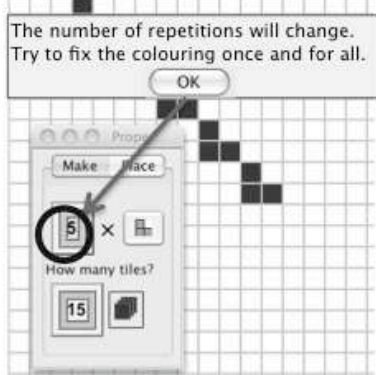

(f)

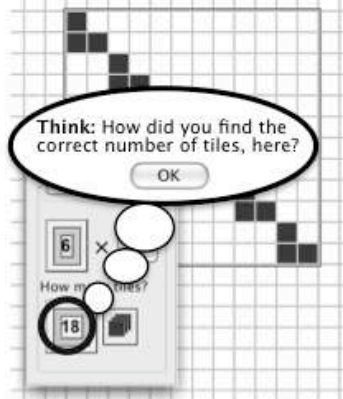

(c)

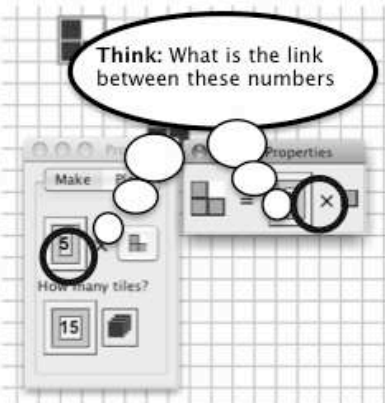

(g)

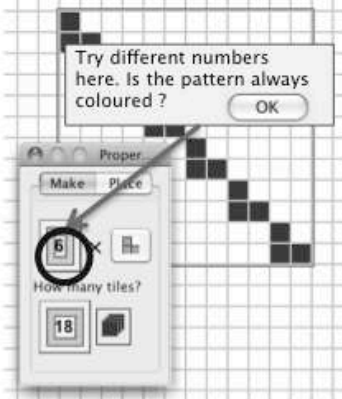

(d)

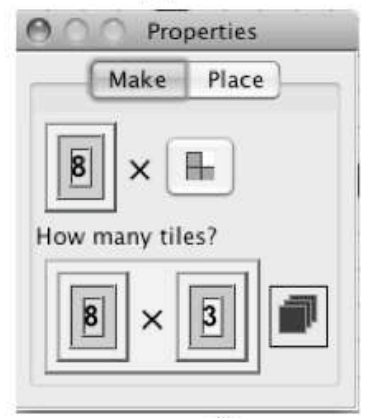

(i)

Figure 6 The messing up strategy as implemented by the intelligent subsystem. (a) Greg unlocks a number. (b) A suggestion reminds him the challenge of colouring the model generally. (c) He repairs the pattern using a specific number - the system generates a reflective prompt. (d) Another suggestion to challenge him colouring generally is provided. (e) He repairs the pattern again - the system generates a prompt to draw his attention to the link between these numbers. (f) Greg realizes that he is not colouring the pattern for any number of building blocks and asks for more help. (g) Before changing his value, the system draws his attention to the two quantities he has to link. (i) Even- tually Greg constructs a general expression

\section{Making a model 'unmessable' - expressing relationships between different patterns}

Being able to express relationships within a pattern explicitly is a large step for some students. However, a further and still more challenging step is to make links between variables. ${ }^{4}$ We illustrate this with a vignette from the work of Conor. He had constructed the model based on two patterns (one made of a building block of two red tiles and another of a building block of three green tiles, see Table 1 and Fig 7). In building these patterns, he had realized the importance of expressing the relationship between the quantities (as explained for the case of Greg).

Conor also knew he had to unlock numbers to make the model animate. He unlocked the number of repeats in both patterns, the 5 for the red pattern and 3 for the green. The system then drew his attention to the General Model, which was messed up. As shown in Fig 7, the 5 had been changed by the system to 8 , and the 3 had been changed to 7 .

Following a similar strategy to Greg's, and with more support from the teacher this time, Conor realized the lack of generality in his model. By changing the value of the unlocked number that repeats the green building block and repairing his model several times, he saw the relationship between the two patterns in his model. Using 
eXpresser's language, he wrote the general rule for the number of red tiles using the variable for the number of repeats in the green pattern (5) as shown in Fig 8.

In the course of the next few sessions, we observed Conor continuing to develop a way of thinking that recognized (1) the need to link patterns together (he even helped other students appreciate this) and (2) the need to express verbally and symbolically the recognized relationships.

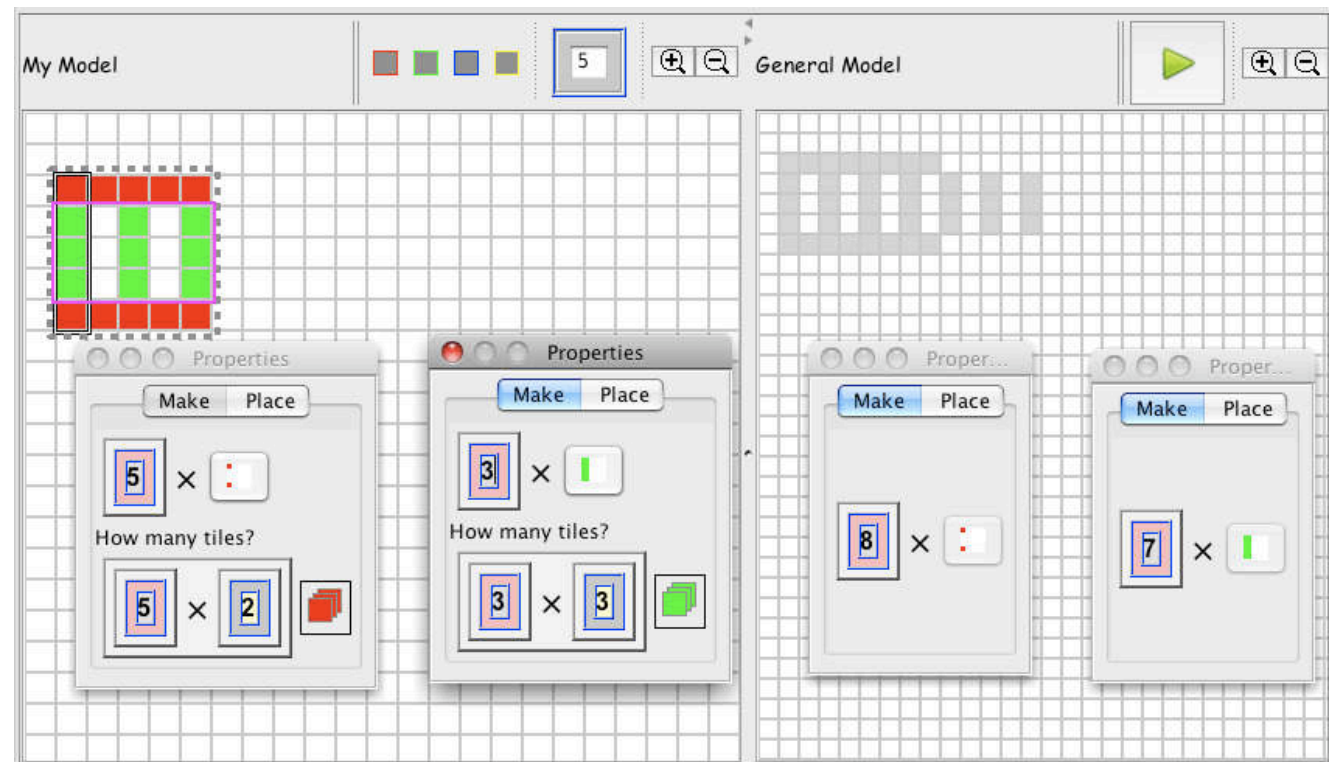

Figure 7 Conor's model is messed up in the General World. He has unlocked both the 5 and the 3 , but has not yet specified an explicit relationship between the two patterns. The General Model is therefore 'messed-up'

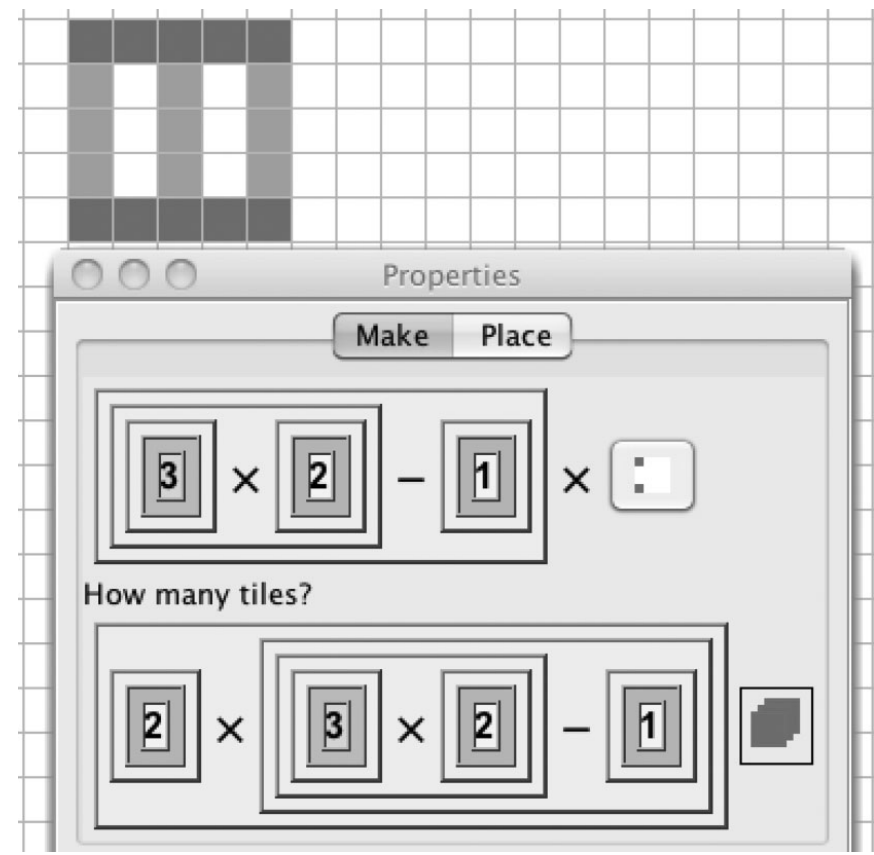

Figure 8 The properties of the red pattern in Conor's final rule. In conventional algebra, the rule for the 
number of red tiles would be written as $(x \times 2-1) \times 2$, where $x$ would represent the number of repetitions of the green pattern

From symbols as generalized numbers to symbols as variables

In terms of our design, the metaphor of locked and unlocked numbers emerged after several attempts to help students express relationships and reach generalizations naturally - or as naturally as the system would allow. We were aware that developing algebraic generalization is facilitated by the availability of an accessible symbol system that was aligned to the construction process. As we interacted with students working with different versions of the microworld, we became increasingly aware of students' difficulties with the ambiguity of the term 'variable' in generalized arithmetic (see Küchemann 1981), and began to look for spontaneous meanings and intuitions emerging from interactions with our system on which we could build, rather than impose the formal concept of variable. After several iterations, we shifted towards the use of symbols as generalized number rather than as a specific unknown or attributes of an object (see Noss et al. 2009, where previous attempts of the same concept are described). In this respect, the value of an unlocked box at any given time acts only as an example of the possible values that it can take. In other words, the box itself becomes a symbol that, as we have seen previously, enables the student to be explicit not only about what changes and what remains the same, but also about perceived relationships within a model (cf. Mason et al. 2005). To assist students to see how the boxes were themselves a kind of algebraic representation, they can give unlocked boxes a name, leading to a closer relationship to the use of letters in algebra either as generalized numbers or as a means of indicating the degree to which a quantity can vary as a result of change in a set of values (cf. Küchemann 1981; Mason et al. 2005). These relatively simple linguistic elements allow students to express themselves quite naturally, emerging as a direct consequence of the model construction, and as an answer to the challenge of colouring the pattern by finding the total number of tiles (see Table 2 for examples). This is exactly the epistemic affordance of the unlocked number symbols and the expressions that can be constructed by using them. By making available to students a language that allows them to express the relationships they perceive, students can begin to experience the power of symbols without having first to master the machinery of conventional notation (Noss \& Hoyles 1996).

How this language became evident in paper-and- pencil questions is exemplified in the work of Fiona. She was asked the question in Fig 9 to find a rule for the number of tiles for the pattern of Fig $3 a$.

She represented the contents of the 'Figure Number' as dots, indicating, in her own words:

$\mathrm{F}$ : It is not just one number, like the 6 that can't change. 
In an attempt to probe her thinking the researcher asked:

R: What do you mean? How many numbers is it?

$\mathrm{F}$ : It is not [possibly meaning many numbers], it is one number but doesn't stay the same.

When she was asked why she provided a name for the number 6 , she said:

$\mathrm{F}$ : Because in a bigger bridge, there will be more ...

$\mathrm{R}$ : What do you mean bigger?

$\mathrm{F}$ : Here [pointing at the horizontal part of the building block, clearly indicating that the bridge top could be longer]

$\mathrm{R}$ : So, this number can change? F: Yes

M: So why not put dots here [pointing at 6 ]?

$\mathrm{R}$ : It is not like this [pointing to the Figure Number], it does not change all the time.

Although Fiona struggled to explain it, she appeared to be expressing another generalization that could be made, one that we did not request: the configuration of the building block could be made out of a variable number of tiles. Moreover, she made a distinction between variables and constants, with a clear rationale for each. She now had a general rule for dealing with that situation as well.

Similarly, Maria's answer in Fig 10 demonstrates that she and other students who responded in similar ways were using the symbols and their grammar as 'objects to think with'.

\section{Conclusion}

This paper describes our attempt to rise to the challenge of creating an exploratory context in which algebraic ways of thinking can be motivated and exploited by relatively young students. To address this challenge, we undertook an epistemological analysis of algebraic generalization and designed a system that addresses its key components, which consists of perceiving and exploiting structure, seeing the general in the particular and expressing generalizations symbolically.

Table 2 Students' models (cf. Table 1) and their corresponding eXpresser and algebraic rules

\begin{tabular}{|c|c|c|}
\hline Student & $\begin{array}{c}\text { Model showing } \\
\text { different constituent } \\
\text { patterns }\end{array}$ & $\begin{array}{c}\text { eXpresser rules and corresponding algebraic } \\
\text { rules }\end{array}$ \\
\hline
\end{tabular}




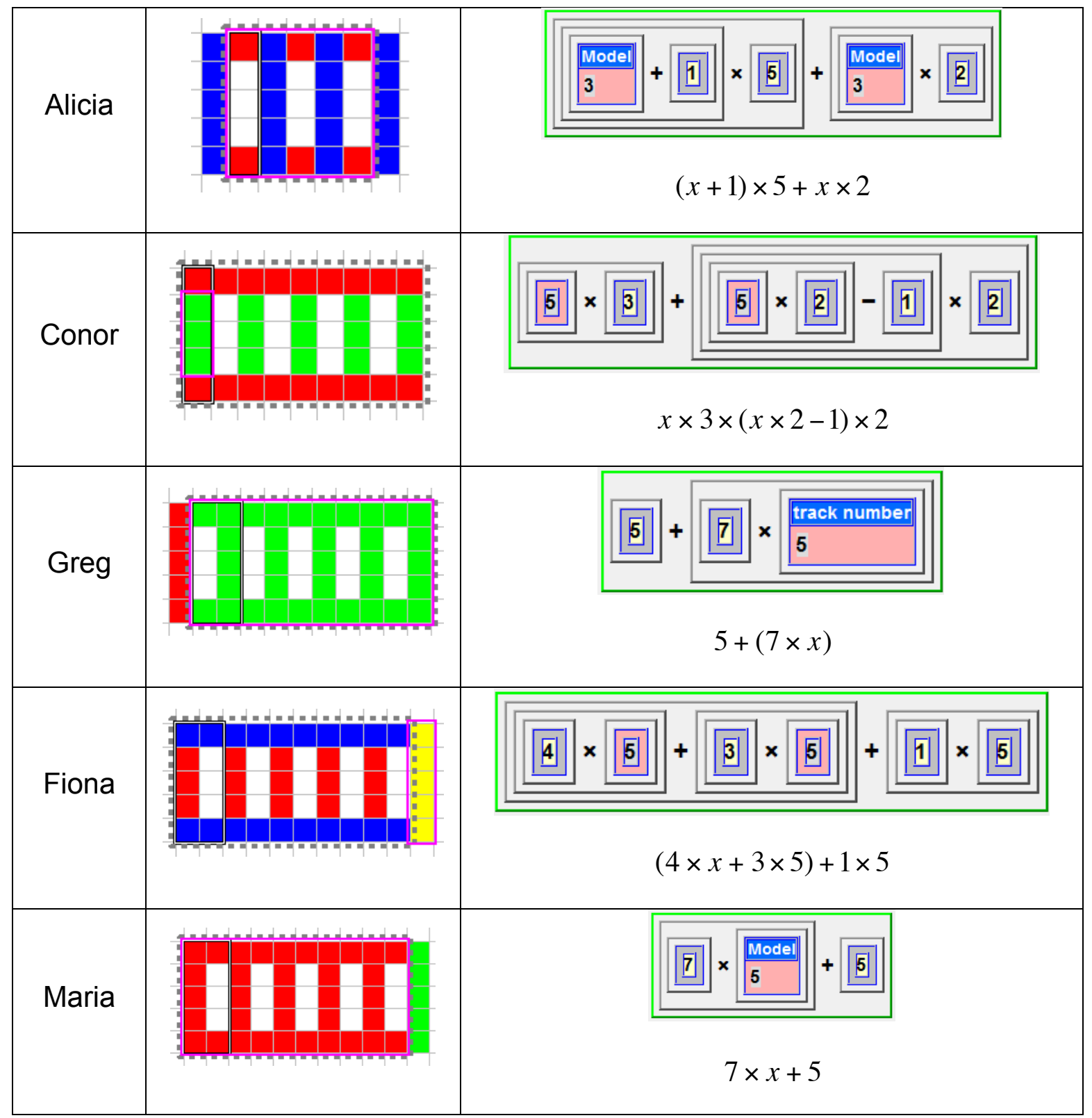

d. Write down a rule for the number of tiles for the pattern for any Figure Number.

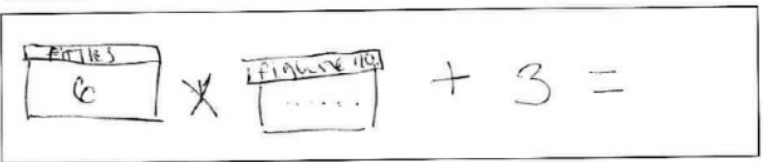

Figure 9 Fiona's rule for the number of tiles for the post-task in Fig $4 a$.

We similarly designed a symbolic language in which putative generalizations could be articulated. 


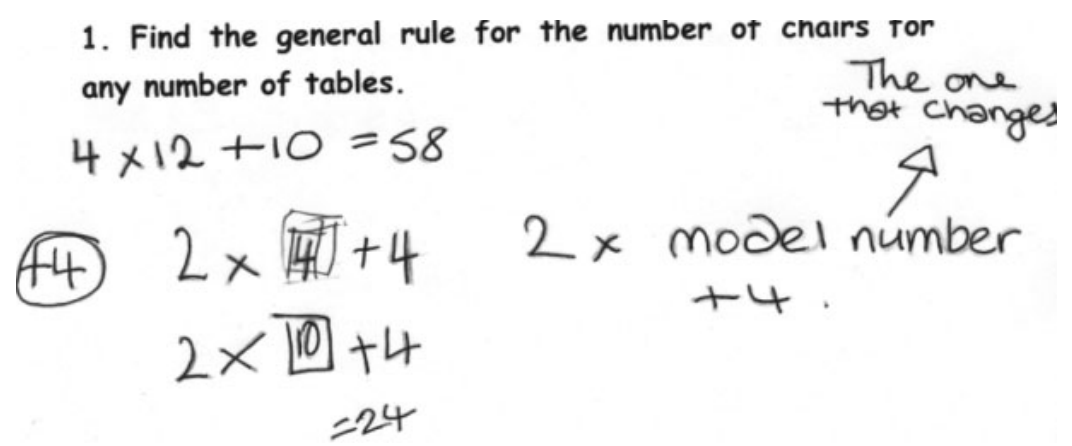

Figure 10 eXpresser's symbols used in answering the post-task in Fig 3b.

Of course, in the iterative design process with which we have engaged so far, we cannot claim to have reached an optimal environment that would, perhaps, be amenable to quantitative evaluation on a large scale. Yet we have reported a satisfactory interim state, one that shows how the MiGen system can support students in developing algebraic ways of thinking, as well as seeing the point of structural reasoning and the need to express relationships explicitly. We do not expect that a few interactions with eXpresser will enable students to master what algebraic generalization requires - this is a process that will continue over many sessions and weeks - or even months and years. However, our aim is that sustained interactions within the microworld sow the seeds for a rationale behind algebraic generalization which can subsequently be used as a framework for learning conventional algebra.

There are two outstanding methodological challenges. The first is to study in depth a cohort of students who use the MiGen system and its successors over a sustained period. The question is whether the task of teaching this cohort conventional algebra is easier, given a shared substrate of experience that can be taken for granted by teacher and students together. This is a challenge that, given the constraints of funded research for such a detailed and longitudinal study, is unlikely to be achievable in the constraints of the MiGen project.

The second challenge is one that we are currently undertaking. The need to support students and teachers in using eXpresser in the classroom is forcing us to formalize further how we might characterize algebraic thinking, in terms of accomplishments, or indicators that students exhibit in interaction with the system (and which the system can recognize). This is not a trivial task. An example of the difficulty has already been dis- cussed in terms of the use of unlocked numbers: we cannot be sure that unlocking numbers (even appropriately) exhibits any 'complete' form of understanding of what is achieved by unlocking. This methodological work will, we hope, be completed when we have a more complete set of correspondences that map student actions onto the specificities of their evolving understandings.

\section{Acknowledgements}

The MiGen project is funded by the ESRC/EPSRC Teaching and Learning Research 
Programme (Technology Enhanced Learning; Award No: RES-139-25-0381). The authors would like to thank the teachers and students who took part in the studies, and acknowledge the contribution of the rest of the members of the inter- disciplinary MiGen team. More details are available at http://www.migen.org.

\section{Notes}

1 The interested reader can interact with eXpresser and the available tutorials at http://www.migen.org.

2 This is specified during construction and can be shown but is hidden in the figure for simplicity.

3 Strictly, there is no such thing as a locked or unlocked number. In eXpresser, numbers appear in boxes. We should refer, therefore, to a locked box in which there is (currently) a specific number whose value is specified and cannot be changed. The clumsiness of this definition is our excuse for committing an abuse of language in referring to unlocking and locking numbers.

4 As we will see, in eXpresser relationships between variables (e.g. the number of repetitions of two different patterns) are expressed by replacing a variable in the pattern by an expression that is a function of a second variable in another pattern.

\section{References}

Becker J. \& Rivera F. (2008) Nature and content of generalization of 7th- and 8th-graders on a task that involves free construction of patterns. In Proceedings of the Joint Meeting of PME 32 and PMENA, Vol. 4 (eds O. Figueras, J. Cortina, S. Alatorre, T. Rojano \& A. Sepulveda), pp. 201-208. Cinvestav-UMSNH, Morelia, Mexico.

Carraher D., Schliemann A. \& Schwartz J. (2008) Early algebra is not the same as algebra early. In Algebra in the Early Years (eds J. Kaput, D. Carraher \& M. Blanton), pp. 235-272. Erlbaum, Mahwah, NJ.

Chua B., Hoyles C., Mavrikis M. \& Geraniou E. (2009) Exploring the link between task features and generalisation. Research in Mathematics Education 11, 73-74.

Cuoco A., Goldenberg E. \& Mark J. (1996) Habits of mind: an organizing principle for mathematics curriculum. Journal of Mathematical Behavior 15, 375-402.

diSessa A. (1995) Epistemology and systems design. In Computers and Exploratory Learning (eds A. diSessa, C. Hoyles, R. Noss \& L. Edwards), pp. 15-29. Springer, Berlin.

Dörfler W. (2008) En route from patterns to algebra: comments and reflections. ZDM 40, 143-160.

Geraniou E., Mavrikis M., Kahn K., Hoyles C. \& Noss R. (2009a) Developing a microworld to support mathematical generalisation. In PME 33: International Group for the Psychology of Mathematics Education, Vol. 3 (eds M. Tzekaki, M. Kaldrimidou \& H. Kaldrimidou), pp. 49-56. Thessaloniki, Greece.

Geraniou E., Mavrikis M., Hoyles C. \& Noss R. (2009b) A learning environment to support mathematical generalisation in the classroom. In CERME 6, Sixth Conference of European Research in Mathematics Education (ed. Weigand), 28 January - 1 February, 2009. Lyon, Frankreich.

Geraniou G., Mavrikis M., Hoyles C. \& Noss R. (2010) Students' justification strategies on equivalence of quasi-algebraic expressions. In Proceedings of the International Conference on Psychology of Mathematics Education, Vol.2 (ed. B. Ubuz), pp. 393-400. Ancara, Turkey. 
Gibson J. (1977) The theory of affordances. In Perceiving, Acting, and Knowing: Toward An Ecological Psychology (eds R. Shaw \& J. Bransford), pp. 67-82. Lawrence Erlbaum, Hillsdale, NJ.

Goldenberg P., Mark J. \& Cuoco A. (2010) An algebraic habits-of-mind perspective on elementary school. Teaching Children Mathematics 16, 548-556.

Greeno J. (1991) Number sense as situated knowing in a conceptual domain. Journal for Research in Mathematics Education 22, 170-218.

Gutierrez-Santos S., Geraniou E., Pearce-Lazard D. \& Poulovassilis A. (2011) Architectural design of teacher assistance tools in an exploratory learning environment for algebraic generalisation. IEEE Transactions of Learning Technologies, in press.

Gutierrez-Santos S., Mavrikis M. \& Magoulas G. (2010) Layered development and evaluation for intelligent support in exploratory environments: the case of microworlds. In Proceedings of Intelligent Tutoring Systems (ITS 2010). Springer Lecture Notes in Computer Science, Vol. 6094 (eds V. Aleven, J. Kay \& J. Mostow), pp. 105-114. Springer Berlin/Heidelberg, Berlin, Heidelberg.

Haverty L., Koedinger K., Klahr D. \& Alibali M. (2000) Solving induction problems in mathematics: not-so-trivial pursuit. Cognitive Science 24, 249-298.

Healy L. \& Hoyles C. (2000) A study of proof conceptions in algebra. Journal for Research in Mathematics Education 31, 396-428.

Healy L., Hoelzl R., Hoyles C. \& Noss R. (1994) Messing up. Micromath 10, 14-17.

Hoyles C. (1993) Microworlds/schoolworlds: the transformation of an innovation. In Learning from Computers: Mathematics Education and Technology. NATO ASI Series. Series F, Computer and Systems Sciences (121) (eds C. Keitel \& K. Ruthven), pp. 1-17. Springer Verlag, Berlin; New York, NY.

Hoyles C. \& Sutherland R. (1989) Logo Mathematics in the Classroom. Routledge, London.

Hoyles C., Noss R. \& Kent P. (2004) On the integration of digital technologies into mathematics classrooms. International Journal of Computers for Mathematical Learning 9, 309-326.

Kieran C. (1989) A perspective on algebraic thinking. In Proceedings of the 13th International Conference for the Psychology of Mathematics Education, Vol. 2 (eds G. Vern, J. Rogalski \& M. Artigue), pp. 163-171. Laboratoire PSYDEE, Paris.

Küchemann D. (1981) Algebra. In Children's Understanding of Mathematics (ed. K. Hart), pp. 102-119. Antony Rowe Publishing Services, London.

Küchemann D. (2008) Looking for Structure: A Report of the Proof Materials Project. Dexter Graphics, London. Küchemann D. (2010) Using patterns generically to see structure. Pedagogies: An International Journal 5, 233-250.

Küchemann D. \& Hoyles C. (2009) From empirical to structural reasoning: tracking changes over time. In Teaching and Learning Proof Across the Grades Hillsdale (eds D.A. Stylianou, M.L. Blanton \& E.J. Knuth), pp. 171-191. Routledge, London; New York.

Lee L. (1996) An initiation into algebraic culture through generalization activities. In Approaches to Algebra: Perspectives for Research and Teaching (eds N. Bednarz, C. Kieran \& L. Lee), pp. 87-106. Kluwer, Dordrecht, the Netherlands.

Lobato J., Ellis A. \& Muñoz R. (2003) How 'focusing phenomena' in the instructional environment afford students' generalizations. Mathematical Thinking and Learning 5, 1-36.

Love, E. (1986) What is algebra? Mathematics Teaching 117, 48-50.

Mason J. (1996) Expressing generality and roots of algebra. In Approaches to Algebra: Perspectives for Research and Teaching (eds N. Bednarz, C. Kieran \& L. Lee), pp. 65-86. Kluwer, Dordrecht, the Netherlands. Mason J., Graham A. \& Wilder S. (2005) Developing Thinking in Algebra. Paul Chapman Publishing, Boca Raton.

Mavrikis M. \& Gutierrez-Santos S. (2010) Not all wizards are from Oz: iterative design of intelligent learning environments by communication capacity tapering. Computers \& Education 54, 641-651.

Mavrikis M., Geraniou E., Noss R. \& Hoyles C. (2008) Revisiting pedagogic strategies for supporting students' 
learning in mathematical microworlds. In Proceedings of the International Workshop on Intelligent Support for Exploratory Environments at EC-TEL'08 (eds M. Mavrikis \& S. Gutierrez-Santos), pp. 41-50. CEUR-WS. org, London.

Noss R. \& Hoyles C. (1996) Windows on Mathematical Meanings: Learning Cultures and Computers. Kluwer Academic Publishers, London.

Noss R., Healy L. \& Hoyles C. (1997) The construction of mathematical meanings: connecting the visual with the symbolic. Educational Studies in Mathematics 33, 203-233.

Noss R., Hoyles C., Mavrikis M., Geraniou E., Gutierrez- Santos S. \& Pearce D. (2009) Broadening the sense of 'dynamic': a microworld to support students' mathematical generalisation. In Special Issue of The International Journal on Mathematics Education (ZDM). Transforming Mathematics Education through the Use of Dynamic Mathematics Technologies, Vol. 41 (eds L. Moreno-Armella \& S. Hegedus), pp. 493-503. Springer Berlin/Heidelberg, Berlin, Heidelberg.

Nunes T. \& Bryant P. (2010) Insights from everyday knowledge for mathematics education. In Innovations in Educational Psychology (eds D. Preiss \& R. Sternberg), pp. 51-78. Springer, New York, NY.

Papert S. (1972) Teaching children to be mathematicians vs. teaching about mathematics. International Journal of Mathematics Education and Science Technology 3, 249-262.

Pellegrino J. \& Glaser R. (1982) Analyzing aptitudes for learning: inductive reasoning. In Advances in Instructional Psychology, Vol. 2 (ed. R. Glaser), pp. 269-345. Lawrence Erlbaum Associates, Hillsdale, NJ.

Pirolli P. \& Greeno J. (1988) The problem space of instructional design. In Intelligent Tutoring Systems: Lessons Learned (eds J. Psotka, L. Massey \& S. Mutter), pp. 181-201. Lawrence Erlbaum Associates, Hillsdale, NJ.

Radford L. (2001) Factual, contextual and symbolic generalizations in Algebra. In Proceedings of the 25th Conference of the International Group for the Psychology of Mathematics Education, Utrecht, The Netherlands, July 12-17, 2001.

Radford L., Bardini C. \& Sabena C. (2006) Rhythm and the grasping of the general. In Proceedings of the 30th Conference of the International Group for the Psychology of Mathematics Education, Vol. 4 (eds $\mathrm{N}$. Jarmila, H. Moraová, M. Krátká \& N. Stehliková), pp. 393-400. Prague, Czech Republic.

Redden T. (1996) Wouldn't it be good if we had a symbol to stand for any number? In Proceedings of the 20th Conference of the International Group for the Psychology of Mathematics Education, Vol. 4 (eds L. Puig \& A. Gutierrez), pp. 195-202. Valencia, Spain.

Rivera F. (2010) Visual templates in pattern generalization activity. Educational Studies in Mathematics 73, 297-328.

Rojano T. (1996) Developing Algebraic Aspects of Problem Solving within a Spreadsheet Environment. Kluwer Academic Publishers, Dordrecht, the Netherlands.

Schoenfeld A. (1985) Mathematical Problem Solving. Academic Press, Orlando, FL.

Sutherland R. \& Rojano T. (1993) A spreadsheet approach to solving algebra problems. Journal of Mathematical Behavior 12, 353-383.

Thompson P. (1987) Mathematical microworlds and intelligent computer-assisted instruction. In Artificial Intelligence and Instruction: Applications and Methods (ed. G. Kearsley), pp. 83-109. Addison-Wesley Longman Publishing Co., Inc, Boston, MA.

Thompson P.W. (1993) Quantitative reasoning, complexity, and additive structures. Educational Studies in Mathematics 25, 165-208.

Trouche L. (2004) Managing the complexity of human/machine interactions in computerized learning environments: guiding students' command process through instrumental orchestrations. International Journal of Computers for Mathematical Learning 9, 281-307. 